A TWO DIMENSIONAL ARMA MODEL FOR THE SIMULATION OF IR BACKGROUNDS

\author{
by \\ Denis F. Strenzwilk \\ Michael P. Meredith, \\ and \\ Walter T. Federer
}

BU-1021-M

April 1989 


\title{
A TWO DIMENSIONAL ARMA MODEL FOR THE SIMULATION OF IR BACKGROUNDS
}

\author{
by \\ Denis F. Strenzwilk \\ Director \\ US Army Ballistic Research Laboratory \\ ATTN: SLCBR-SE-W \\ Aberdeen Proving Ground, Maryland 21005-5066, USA \\ Michael P. Meredith ${ }^{+}$ \\ and \\ Walter T. Federer \\ Mathematical Sciences Institute \\ 337 Warren Hall \\ Cornell University \\ Ithaca, NY 14853-7801, USA
}

Key Words and Phrases: 2D ARMA Model; IR Backgrounds; clutter; simulation; time series; spectrum; spatial correlation; spatial moving average.

\begin{abstract}
At the US Army Ballistic Research Laboratory (BRL), Aberdeen Proving Ground, Md., weapon system analysts use background models in order to: 1) establish 'clutter' thresholds for firing algorithms: and, 2) to study the masking and false alarm effect of background in their effort to evaluate the performance of various weapon systems. The BRL has received from US Army Engineer Waterways Experiment Station (WES) several large data bases comprised of blackbody
\end{abstract}


temperatures derived from measurements obtained with an IR sensor. The sensor was mounted on a helicopter and scanned in the cross-track direction perpendicular to the direction of flight (in-track). The data consist of temperatures of scene elements (pixels) for a plowed field, a forested area, and a grassy field. The primary objective of this research is to provide a simple mathematical model which provides simulated data that are consistent with descriptive statistics from the original spatially correlated data base. Such statistics include the mean and standard deviation of temperature, and its 'energy spectrum'. The Mathematical Sciences Institute (MSI) at Cornell University have suggested time series models and a Spatial Moving Average (SMA) model as two approaches to the problem. One long term objective of this type of investigation is to construct a method for relating parameters in the model to physical constants. If successful, the model may then be extended over the diurnal cycle and seasons.

\section{INTRODUCTION}

BRL to date has modeled target signatures in a deterministic manner while background signatures have been treated stochastically. The deterministic model for target signatures is appropriate because under a particular set of conditions, the signature is rather well defined and is amenable to a single characterization. The case is not the same for backgrounds, which are many and varied. Thus, the general approach in modeling backgrounds has been to select a data set of a homogeneous scene, to extract pertinent statistics, such as, the mean temperature, the standard deviation, the 'energy spectrum', the correlation between pixels, etc., and finally, to develop a model, which can simulate a 'typical' background segment with these same statistics.

Botkin et al. (1981) developed a model for which the radiometric characteristics of a region of the same texture type were modeled statistically as a random variable with a Gaussian amplitude distribution and an exponential spatial autocorrelation function. The radiance data, which they analyzed, could also be expressed as an equivalent blackbody temperature as were the data in this paper. The mean radiance, the standard deviation of the radiance amplitude values, and a characteristic length defining the spatial extent of the correlation function are the underlying statistical parameters, which are generally different for each texture type. Therefore, the model of a multi-texture background scene was described as a non-stationary Markov 
process.

Another model proposed by Rapp (1983) assumes that in a given row the background pixels have a temperature drawn from a normal distribution with a given mean and variance, in which the temperatures are arranged spatially in monotonic increasing or decreasing order according to an interval length governed by an exponential distribution. The correlation length in the exponential distribution is chosen in such a way that the energy spectrum of the model matches the one for the measured data. Adjacent rows are the same except for a lateral shift by one element. This model was developed further by Rapp (1984) by first selecting the temperature values for the individual ground resolution elements in a row from a normal distribution, and then processing them through a low-pass spatial filter to generate a new string of values, whose neighboring elements are correlated in a specific manner. Then, he proceeded as before to get a two dimensional array of temperatures.

Later, Rapp and Durfee (1987) developed a different model, where a two dimensional array of uncorrelated temperatures were drawn from a normal distribution with the desired mean temperature and a variance, which was some multiple of the desired variance. Then, a $N x N$ template was constructed and the temperature of each element was replaced by the average temperature of this moving window. This procedure was repeated for all the elements. The size of the template was adjusted until the energy spectrum of the model matched the one for the measured data.

In most smart weapon simulations, the sensor scans across many square meters of background before any target is encountered. During this time, the sensor's signals are processed by a target discrimination circuit that usually includes some sort of adaptive threshold logic. Usually for this type of discrimination, the signal's Root-Mean-Square (RMS) average is developed as a measure of background 'clutter'. Target detections occur when the instantaneous sensor output exceeds a threshold value that is proportional to the average of the output signal. The sensor's output signals produced by scanning the modeled background are thus used to provide a basis for setting the detection threshold; this is perhaps the most important function of the background. The stochastic background modeling approach currently being used at the BRL is based on a normal temperature assumption. It is quite well suited to provide a reasonable estimate of average clutter in many situations, even though the temperature distribution of the pixels is not normal. However, a background model also ought to include some provision for sources of false detection. The simple stochastic background model described here is clearly not capable of fulfilling this objective, for there is only a very remote 
possibility of a false alarm when the detection threshold is set to some multiple of the RMS signal. What is lacking is a means for incorporating some realistic scene features that would constitute possible sources for false alarms.

Given that a target signature model with a reasonable degree of fidelity is mated with a valid stochastic background signature model, it is possible to predict when and where a target detection is likely to occur. Probabilities of target detection can be inferred and the sensor/processor may be analyzed in terms of performance given a target encounter. This has been the BRL approach for many smart weapon simulations. A different approach must be taken if one wants to make some assessment of the smart weapon's capability for rejecting false targets. Ideally, the background infrared signature model used for this type of performance analysis ought to include a realistic characterization of individual scene elements that might confuse the target discrimination logic, like the transition region between different texture types. BRL felt it was possible to develop a better background signature model than was currently in use, which was predictive in nature and includes specific features that are potential false targets. The first thoughts of Strenzwilk, Meredith, and Federer (1988) on this model were expressed in the form of a clinical presentation at the University of Delaware from which this paper originated.

Up to this point the discussion has been confined to simple scenes, e.g., a grassy field, a plowed field, a forested area, etc. Once a suitable model for a simple scene has been developed, BRL wants to construct arbitrary scenes from these simple scenes. Thus a forested area of any desired size may be placed next to a plowed field. A road may be added to the scene. This compound scene with these three different kinds of textures could then be used in computer simulations of smart weapon concepts. All kinds of different compound scenes of arbitrary geometry and composition could be constructed from the models of the simple scenes. Thus the ability to construct compound scenes from simple scenes is a desideratum of the modeling effort.

\section{DATA BASE}

An alternative to "modeling" the background signatures either deterministically or stochastically would be to use actual scene measurements as inputs to the smart weapon sensor model. This would require that the measured background signatures be compatible with the sensor model in terms of viewing direction, detector wavelength band, and scene pixel size. Although the existing infrared background signature data base is rather 
extensive, very few of these sources have the requisite characteristics for smart weapons system evaluations that are currently being conducted. One source of data found to be generally compatible with the type of smart weapons that are being investigated at the BRL is the set of infrared scanner measurements of a rural area near Hunfeld, Germany, made by the US Army Engineer Waterways Experiment Station (WES). For these measurements WES employed a helicopter-mounted Daedalus infrared scanner operating in the wavelength band of 8.5 to 12.5 micrometers. The scanner was flown over the test terrain at altitudes of 200 and 600 feet. The 600 -feet data were taken on 9 September 1982 at 10:15 AM, while the 200-feet data were taken on 10 September 1982 at 12:34 PM. The temperature of the air ( $15 \mathrm{~cm}$ above the ground ) was $20^{\circ} \mathrm{C}$ on 9 September and $28^{\circ} \mathrm{C}$ on 10 September.The sizes of the corresponding ground resolution elements were roughly compatible with the 0.1 meter resolution that is optimum for the BRL's smart munition evaluation efforts, and the site of the measurements and the scene content is quite appropriate. The advantage of modeling this data set is that the model can be checked against the actual data in the simulation of a smart weapons concept.

In this paper the time series models were applied to the data of the forested area. The data of the plowed field and grassy area have a similar format. The data base for the forested area is composed of 250 rows of temperatures. Each row contains 500 temperature pixels. Thus, for this data set there are 250 rows times 500 columns or 125,000 pixels of temperature. A row of data (500 pixels) represents one 'cross-track' scan of the sensor, which was mounted on a helicopter that flew in a direction perpendicular to the rows ('in-track') . After processing the data with ground truth information, it was concluded that at the $600 \mathrm{ft}$ altitude the in-track (flight direction) dimension of the pixels was $0.3050 \mathrm{~m}$ whereas the cross-track dimension was $0.1525 \mathrm{~m}$. The data are highly correlated both in-track and cross-track.

\section{TIME SERIES MODEL}

Several autoregressive moving average models, $\operatorname{ARMA}(p, q)$, were fitted to the data. It was decided that for each row of 500 observations a $\operatorname{ARMA}(1,1)$ was the model which best fit the data. If the actual temperature observation was used to forecast the next pixel value for a complete row of simulated data, the forecasted data had the same spatial pattern and statistical characteristics as the actual data. If, however, the forecasted value was used to forecast the next pixel value in the row, the resulting set of forecasted values did not have the same pattern but did have the same characteristics. Thus, to preserve the spatial pattern in the time series approach, the actual data base would have to 
be used to make the forecasts. It was decided that for most applications it would suffice to have a model with the same statistical characteristics. Therefore, the actual observation of the temperature of the first pixel in each row was used to forecast the 2 nd value and thereafter the forecasted value was used to forecast the next pixel value in the row. The ARMA used was

$$
\tilde{z}_{t}=\phi_{1} \tilde{z}_{t-1}-\theta_{1} a_{t-1}+a_{t}\left(\mu_{a}, \sigma_{a}\right),
$$

III.1

where

$t$ equals $1,2,3, \ldots, 500$

$z_{t}$ temperature of $t$ th pixel in row

$\tilde{z}_{t} \quad$ temperature of $t$ th pixel in row minus the mean, $\left(z_{t}-\mu\right)$

$\mu \quad$ mean temperature of row

$\phi_{1}$ autoregressive parameter of order one

$\theta_{1}$ moving average parameter of order one

$a_{t}$ random number for $\mathrm{t}$ th pixel from $N\left(\mu_{a}, \sigma_{a}^{2}\right)$, called residual or 'shock'

$\mu_{a} \quad$ mean temperature of residuals

$\sigma_{a} \quad$ standard deviation of residuals

\section{ENERGY SPECTRUM}

Let us represent the the two dimensional array of temperatures as a matrix, whose elements $T(l, m)$ are

$$
T(l, m)=z_{t}^{l},
$$

where

$z_{t}^{l} \quad$ is the value of $z_{t}$ in the lth row

m equals $0,1,2, \ldots, N_{r}-1$

$N_{r}$ is the number of pixels in a row $(=500)$

1 equals $0,1,2, \ldots, N_{c}-1$

$N_{c}$ is the number of pixels in a column $(=250)$.

$t$ equals $m+1$

The discrete Fourier transform (DFT) for a row of temperatures is

$$
Z^{l}(k)=\sum_{m=0}^{N_{r}-1}\left(T(l, m)-\mu^{l}\right) \exp \left[-i\left(2 \pi / N_{r}\right) m k\right],
$$

where

$k$ equals $0,1,2, \ldots, N_{r}-1$,

$\mu^{l}$ mean temperature of $l$ th row 
and for a column of temperatures is

$$
Z_{m}(k)=\sum_{l=0}^{N_{c}-1}\left(T(l, m)-\mu_{m}\right) \exp \left[-i\left(2 \pi / N_{c}\right) l k\right]
$$

where

$k$ equals $0,1,2, \ldots, N_{c}-1$.

$\mu_{m}$ mean temperature of $m$ th column

The frequency of a row $f_{r}$ is

$$
f_{r}=m / N_{r} \Delta_{r}
$$

IV.4

where $\Delta_{r}$ is $.1525 \mathrm{~m}$, and the frequency of a column $f_{c}$ is

$$
f_{c}=l / N_{c} \Delta_{c}
$$

where $\Delta_{c}$ is $.3050 \mathrm{~m}$. The energy of the kth frequency in the lth row $S^{l}(k)$ is

$$
S^{l}(k)=Z^{l}(k) Z^{l^{*}}(k)
$$

and the energy of the kth frequency in the $m$ th column $S_{m}$ is

$$
S_{m}(k)=Z_{m}(k) Z_{m}^{*}(k),
$$

where the symbol * denotes the complex conjugate. The cross-track energy spectrum and the in-track energy spectrum are a statistical measure of the correlation of the data, and result when $S^{l}(k)$ or $S_{m}(k)$ are plotted against frequency, respectively. (Zero frequency is excluded as the interest is in the the variation from the mean.)

The energy spectrum is symmetrical about the Nyquist frequency, which occurs at $f_{r}=.5 / \Delta_{r}=3.279$ cycles per metre and at $f_{c}=.5 / \Delta_{c}=1.639$ cycles per metre. Thus, it is common practice to multiply the energy of the $k t h$ frequency by a factor of two, and to plot the energy spectrum up to the Nyquist frequency. This convention was used in this paper.

It is well known that the energy spectrum for a row or column of $N$ samples can be rewritten in terms of the sample autocovariances $\left(c_{j}\right)$ as

$$
\frac{S(k)}{N}=c_{0}+2 \sum c_{j=1}^{N-1} \cos [(2 \pi / N) j k]
$$

where

$$
c_{j}=\frac{1}{N} \sum_{m=0}^{N-1-j}\left(T_{m}-\mu\right)\left(T_{m+j}-\mu\right),
$$


where according to Box and Jenkins (1976) the most satisfactory estimate of the $j$ th lag autocorrelation $\rho_{j}$ is given by

$$
r_{j}=\frac{c_{j}}{c_{0}},
$$

provided that $j$ does not exceed $N / 4$. The correlation distance is that distance at which the autocorrelation function falls to the value of $1 / e$, and it plays an important role in the other models, which were summarized in the introduction.

In order to approximate an ensemble average by a spatial average, it is customary according to La Rocca and Witte (1980) to average $S^{l}(k)$ over the 250 rows and to average $S_{m}(k)$ over the 500 columns. Thus, the average energy of the $k t h$ frequency of the 250 rows $S^{r}(k)$ is

$$
S^{r}=(1 / 250) \sum_{l=0}^{249} S^{l}(k),
$$

and the average energy of the $k t h$ frequency of the 500 columns $S_{c}(k)$ is

$$
S_{c}=(1 / 500) \sum_{m=0}^{499} S_{m}(k) .
$$

\section{TWO DIMENSIONAL ARMA MODEL}

The criterion for selecting a model was that its mean temperature, its standard deviation, and its energy spectrum, which measures the correlation in the temperature, be in good agreement with the data. The mean temperature and the standard deviation of the data were evaluated. The energy spectrum of the data was evaluated and plotted versus the frequency for the cross-track and in-track directions.

The first two dimensional (2D) model tried was to simulate the 250 rows of temperature by using Equation (III.1) and the appropriate parameter estimates for each row. The mean temperature and its standard deviation were in good agreement. The cross-track energy spectrum for the rows $S^{r}(k)$ was also in good agreement with the data since the ARMA model, which gives the best estimate of the autocorrelation function, was fitted to the rows. However, the in-track energy spectrum for the columns $S_{c}(k)$ was not in agreement with the data. This was expected because nothing had been done to introduce correlation between adjacent rows, which is a consequence of the spatial pattern not being preserved. Several approaches based on using the 
temperatures in the row above to forecast the next forecast in the row below were suggested as a way of introducing correlation. None of these approaches were successful.

After inspection of the spatial temperature variation of several sets of adjacent rows, some trends were noticed. The first was that $T(l, m)$ and $T(l+1, m)$ had similar values and the second was that if $T(l, m+1)$ increased or decreased from $T(l, m)$, then $T(l+1, m+1)$ would show a similar increase or decrease from $T(l+1, m)$. Perhaps, the shock $a_{t}^{l}$ that produced $T(l, m+1)$ was correlated with the shock $a_{t}^{l+1}$ that produced $T(l+1, m+1)$. Based on this physical evidence, the assumption was made that $a_{t}^{l}$ was related to $a_{t}^{l+1}$ through a bivariate normal distribution $g\left(a_{t}^{l}, a_{t}^{l+1}\right)$ given by

$$
g\left(a_{t}^{l}, a_{t}^{l+1}\right)=\left(\frac{1}{2 \pi \sigma_{a}^{l} \sigma_{a}^{l+1} \sqrt{1-\rho^{2}}}\right) \exp \left[-\frac{1}{2\left(1-\rho^{2}\right)}\left(\left(\frac{a_{t}^{l}}{\sigma_{a}^{l}}\right)^{2}-2 \rho \frac{a_{t}^{l} a_{t}^{l+1}}{\sigma_{a}^{l} \sigma_{a}^{l+1}}+\left(\frac{a_{t}^{l+1}}{\sigma_{a}^{l+1}}\right)\right)\right], \mathrm{V} .1
$$

where the means of the residuals $\mu_{a}^{l}$ do not appear since they nearly zero, and the correlation coefficient $\rho$ has the range

$$
-1<\rho<+1 \text {. }
$$

The marginal probability density function (pdf) for $a_{t}^{l}$ is

$$
g_{1}\left(a_{t}^{l}\right)=N\left(0,\left(\sigma_{a}^{l}\right)^{2}\right)
$$

and the marginal pdf for $a_{t}^{l+1}$ is

$$
g_{1}\left(a_{t}^{l+1}\right)=N\left(0,\left(\sigma_{a}^{l+1}\right)^{2}\right) .
$$

The conditional distribution for $a_{t}^{l+1}$ given $a_{t}^{l}$ is

$$
g_{2}\left(a_{t}^{l+1} / a_{t}^{l}\right)=N\left(\rho\left(\frac{\sigma_{a}^{l+1}}{\sigma_{a}^{l}}\right) a_{t}^{l},\left(\sigma_{a}^{l+1}\right)^{2}\left(1-\rho^{2}\right)\right)
$$

Now, the following procedure was used to find that value of $\rho$ which minimized, in the least squares sense, the difference between the in-track energy spectrum of the data $S_{c}(k)$ and the in-track energy spectrum of the simulated data $S_{c}(k ; \rho)$. For a given value of $\rho$ the first row of simulated temperatures was generated from the ARMA model given in Equation (III.1) with the appropriate parameter estimates by using the values of $a_{t}^{0}$ drawn from 
the marginal distribution given in Equation (V.3). The second row of simulated temperatures was generated from the ARMA model given in Equation (III.1) with the appropriate parameter estimates by using the values of $a_{t}^{1}$ drawn from

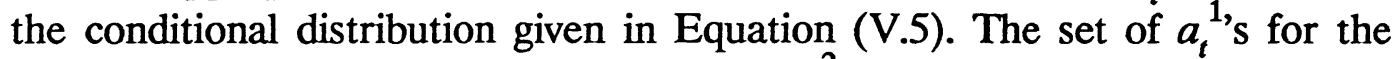
second row were then used to generate the $a_{t}^{2} s$ for the third row through the conditional distribution given in Equation (V.5), etc., until 250 rows of simulated temperatures were generated. Then, the in-track energy spectrum $S_{c}(k ; \rho)$ was evaluated, and the sum of squared differences between the intrack energy spectrum for the observed data and the simulated data was evaluated for that value of $\rho$. This process was repeated in a systematic way for several values of $\rho$. The value of $\rho$, minimizing this sum of squares, was chosen for use in the predictive model.

If $\varepsilon(l, t)$ is taken to be a white noise spatial series, whose elements have zero mean and variance 1 , the shocks can be expressed as

$$
a_{t}^{0}=\sigma_{a}^{0} \varepsilon(0, t), \quad l=0
$$

and

$$
a_{t}^{l}=\left(1-\rho^{2}\right)^{1 / 2} \sigma_{a}^{l} \varepsilon(l, t)+\rho\left(\frac{\sigma_{a}^{l}}{\sigma_{a}^{l-1}}\right) a_{t}^{l-1} \cdot \quad l \neq 0
$$

By repeated use of Equation (V.7) along with Equation (V.6),

$$
a_{t}^{l}=\left(\sigma_{a}^{l}\right)\left(1-\rho^{2}\right)^{1 / 2} \sum_{i=0}^{l-1} \rho^{i} \varepsilon(l-i, t)+\left(\sigma_{a}^{l}\right) \rho^{l} \varepsilon(0, t) . \quad l \neq 0
$$

Thus, this 2D ARMA(1,1) model can be expressed as

$$
\tilde{T}(l, t)=\phi_{1}^{l} \tilde{T}(l, t-1)-\theta_{1}^{l} a_{t-1}^{l}+a_{t}^{l},
$$

where $\tilde{T}(l, t)=T(l, t)-\mu^{l}$. The constant coefficients of the finite set of spatial coordinate observations of the temperature and of their white noise have the generalized form of other 2D ARMA models, but with the important difference that their values vary with row number. It would be very interesting to compare this 2D ARMA model with the one of Cadzow and Ogino (1981) if the formidable task of fitting their model to this $(250 \times 500)$ array of temperatures were carried out.

Equally instructive was to write $\tilde{T}(l, t)$ in terms of the $a_{t}^{l, \mathrm{~s}}$, ( $\psi$ weights), and then in terms of the $\varepsilon(l, t)$ 's and $\rho$ by using Equations (V.6) and (V.8). After a modicum of algebra, the expected spectrum for the in-track direction 
can be evaluated.If $\alpha(l, j)$ is defined as

$$
\alpha(l, j) \equiv \frac{\sigma^{l} \sigma^{l+j}\left[1-\phi_{1}^{l} \theta_{1}^{l+j}-\theta_{1}^{l} \phi_{1}^{l+j}+\theta_{1}^{l} \theta_{1}^{l+j}\right]}{\left(1-\phi_{1}^{l} \phi_{1}^{l+j}\right)},
$$

where the $l$ and $l+j$ superscripts indicate the row with which the parameter is associated. Then, the expected value of $c_{j}$ for the $m t h$ column (in-track direction) is

$$
E\left(c_{j}\right)=\frac{1}{250} \sum_{l=0}^{249-j}\left[\mu^{l} \mu^{l+j}-\mu_{m} \mu^{l}-\mu_{m} \mu^{l+j}+\mu_{m}^{2}+\alpha(l, j) \rho^{j}\right],
$$

where the mean temperature of the $m$ th column is $\mu_{m}$ and $\rho^{j}$ is $\rho$ raised to the $j$ th power. When the values averaged over the 500 columns of $\bar{\mu}_{m}=13.1^{\circ} \mathrm{C}$ and $\frac{\mu_{m}}{\mu_{m}}=170.8^{\circ} \mathrm{C}$ are substituted for $\mu_{m}$ and $\mu_{m}^{2}$ into Equation (V.11), the analytical form of the expected spectrum can be obtained by substitution into Equation (IV.8). Now, the sum of squared differences between the in-track energy spectrum for the observed data and the expected spectrum was evaluated, and minimized as a function of $\rho$.

If the following definitions are used

$$
\gamma_{0}^{l} \equiv \frac{\left(\sigma_{a}^{l}\right)^{2}\left[1-2 \theta_{1}^{l} \phi_{1}^{l}+\left(\theta_{1}^{l}\right)^{2}\right]}{\left(1-\left(\phi_{1}^{l}\right)^{2}\right)},
$$

and

$$
\gamma_{1}^{l} \equiv \frac{\left(\sigma_{a}^{l}\right)^{2}\left[\left(1-\theta_{1}^{l} \phi_{1}^{l}\right)\left(\phi_{1}^{l}-\theta_{1}^{l}\right)\right]}{\left(1-\left(\phi_{1}^{l}\right)^{2}\right)},
$$

then, the expected value of $c_{j}$ for the lth row (cross-track direction) is

$$
E\left(c_{0}\right)=\gamma_{0}^{l},
$$

and

$$
E\left(c_{j}\right)=\frac{(500-j)\left(\phi_{1}^{l}\right)^{j-1} \gamma_{1}^{l}}{500} .
$$

When these expected values of $c_{j}$ are averaged over the 250 rows and substituted into Equation (IV.8), the analytical form of the expected spectrum in the cross-track direction can be obtained. 


\section{CONCLUSIONS FOR 2D ARMA MODEL}

The value of $\rho$ which minimized the difference in the actual and simulated in-track energy spectrum was 0.89 , whereas the one which minimized the difference in the actual and expected in-track energy spectrum was 0.76 . The expected in-track energy spectrum has a non zero value of $16.0^{\circ} C^{2}$ at $k=0$, whereas each column of the simulated in-track energy spectrum has a value of zero at $k=0$. When the simulated energy spectrum is averaged over the 500 columns, it approximates the expected spectrum at all values of $k \neq 0$. Thus, the value of $\rho$, which minimized the difference in the actual and expected energy spectrum, ( $k=0$ values excluded), is 0.81 . A good discussion of the spectral differences between a realization of a stochastic process (deterministic) and the stochastic process itself is given by Jenkins and Watts (1968). The mean temperature $\bar{T}$ of the data base was $13.1^{\circ} C$ and its standard deviation $\sigma$ was $1.2^{\circ} \mathrm{C}$, whereas the simulated data base had a mean temperature of $13.1^{\circ} \mathrm{C}$ and a standard deviation of $1.1^{\circ} \mathrm{C}$. The comparison of the cross-track energy spectrum for the data, for the simulated data, and expected data $(k=0$ value included) can be seen in Figure 1. Similarly, the comparison of the in-track energy spectrum for the data, for the simulated data, and expected data $(k=0$ value included) can be seen in Figure 2. The agreement in both cases is good. Thus, this two dimensional ARMA model can simulate the statistical characteristics of the data, but not the spatial variations. Furthermore, to obtain more than 250 rows use Row 249 parameter estimates for Row 251, Row 248 parameter estimates for Row 252, etc., and essentially form a mirror image of the original 250 rows. To make rows longer, just draw more than 500 shocks for each row. An alternative to this procedure would be to use the $250 \mathrm{x}$ 500 array of temperatures as the basic unit and extend it in any direction by mirror reflection.

One untried approach to improve this 2D ARMA model would be to take the average value of the ARMA parameter estimates for the 250 rows or at least several consecutive rows to obtain "representative parameter estimates". Then, randomly perturb these representative parameter estimates within their observed bounds for each row to be simulated, and proceed as before to determine a suitable value of $\rho$ for the simulated temperatures.

Another untried approach to improve this 2D ARMA model might be to fit an ARMA model to every kth row of data. Use the appropriate parameter estimates for Rows $1, k+1,2 k+1$,etc. . For the rows in between 1 and $k$, use a weighted average for the parameter estimates, e.g., Row 2 values are $[(k-1) / k]$ (value of Row 1$)+(1 / k)$ (value of Row $k$ ), Row 3 values are $[(k-2) / k]($ value of Row 1$)+(2 / k)$ (value of Row $\mathrm{k})$, etc. (Note that a small 
amount of noise could be added to each value.) Proceed as before to determine a suitable value of $\rho$ for the simulated temperatures.

\section{SPATIAL MOVING AVERAGE MODEL}

The model described in this section differs from the ARMA models discussed above in that it is a two-dimensional model from the start whereas the others are one-dimensional models adjusted to give a two-dimensional array of spatially correlated observations. It also offers more promise of reproducing the spatial variation of the data, but at present it has not been applied to our problem. The steps for the SMA model are:

1. Generate an array of $Z_{i j}$, which are independent, identically distributed normal random variables, $\operatorname{NIID}\left(0, \sigma^{2}\right)$.

2. Use $Z_{i j}$ in a spatial moving average (SMA) to construct the temperature datum $T_{n, m}$ as

$$
T_{n, m}=\bar{T}+\sum_{i=-p}^{p} \sum_{j=-q}^{q} A_{i j} Z_{n+i, m+j},
$$

where $E\left[T_{n, m}\right]=\bar{T}$, and

$$
\begin{aligned}
& \operatorname{Cov}\left(T_{n, m}, T_{n+s, m+t}\right)=0 \text {, if }|s|>p,|t|>q ; \\
& \operatorname{Cov}\left(T_{n, m}, T_{n+s, m+t}\right)=\sigma^{2} \sum^{p} \quad \stackrel{q}{\Sigma} A_{i j}^{2} \text {, if } s=0, t=0 ; \quad \text { VII.2.b } \\
& i=-p \quad j=-q
\end{aligned}
$$

and

$$
\operatorname{Cov}\left(T_{n, m}, T_{n+s, m+t}\right)=\sigma^{2} \sum_{i=-p+s}^{p} \underset{j=-q+t}{\sum} A_{i j} A_{i-s, j-t} \text {, otherwise. VII.2.c }
$$

3. $A_{i j}$ are chosen by the researcher such that

$$
\sum_{i j} \sum A_{i j}=1
$$

Table 1 illustrates the needed coefficients $A_{i j}$ for $p=1, q=1$ that multiply the random variable $Z_{n m}$ in order to obtain a value for $T_{n, m}$ in Equation (VII.1). Some $A_{i j}$ may be chosen to be zero or some other value. Problems concerning the optimal determination of $A_{i j}$ in SMA in order to match the energy spectrum are discussed by Sharma and Chellapa (1986). 
TABLE 1. Coefficients of the Spatial Moving Average for Constructing the Datum $T_{n, m}$ Using the NIID Random Variables $Z_{i j}$.

\begin{tabular}{c|c|c|c|}
\multicolumn{1}{c}{} & \multicolumn{1}{c}{$\mathrm{m}-1$} & \multicolumn{1}{c}{$\mathrm{m}$} & \multicolumn{1}{c}{$\mathrm{m}+1$} \\
\cline { 2 - 4 } $\mathrm{n}-1$ & $A_{-1,-1}$ & $A_{-1,0}$ & $A_{-1,1}$ \\
\cline { 3 - 4 } $\mathrm{n}$ & $A_{0,-1}$ & $A_{0,0}$ & $A_{0,1}$ \\
\cline { 3 - 4 } $\mathrm{n}+1$ & $A_{1,-1}$ & $A_{1,0}$ & $A_{1,1}$ \\
\hline
\end{tabular}

\section{SOME COMMENTS}

Our primary objective in this research was to provide a simple mathematical model which provides simulated data that are consistent with descriptive statistics from the original spatially correlated data base. Our 2D ARMA model met our criterion that its mean temperature, its standard deviation, and its energy spectrum, which measures the correlation in the temperature, be in good agreement with the data, even though it did not reproduce the spatial variation in the data. Our assumption that the shocks in adjacent rows be drawn from a bivariate normal distribution was the ingredient that introduced the necessary two dimensional spatial correlation in the simulated data. Some additional approaches for simplifying our 2D ARMA model, which were centered around reducing the number of ARMA parameter estimates needed for simulation, have been suggested. In addition a spatial moving average model has been outlined as an alternative method for this problem.

Our 2D ARMA model is an improvement over the models based on the normal temperature assumption that are currently being used at the BRL, especially since the time series approach naturally forecasts outlier temperatures ( false alarms ) that are found in the data. In time, after more data are analyzed by ARMA models, methods for relating the parameter estimates to physical constants will be found. If successful, the model may then 
be extended over the diurnal cycle and seasons. Also, for the theorists, an ndimensional spatially correlated model is easily constructed.

\section{ACKNOWLEDGEMENTS}

Garl L. Durfee of BRL is thanked for processing the original WES data that was analyzed in this research. Monte Coleman of BRL is acknowledged for setting up procedures for the control language necessary to run the BMDP Statistical Software time series package on the CDC Cyber computer. Barbara Broome and Ken Joel, both of BRL, gave expert advise on the computer graphics.

\section{BIBLIOGRAPHY}

+ present address: Miami Valley Laboratories, The Procter \& Gamble Co., P.O. Box 398707, Cincinnati, Ohio 45239.

Botkin, E., Kelley, G., Gawronski, G., Krassner, J., Klop, M., Goldman, R., Baird, A., and D'Argenio, C., "Infrared Modeling and Analyses (IRMA), Volume I:Scene Generation and Sensor/Seeker Interface," AFATL-TR81-65, 31 August 1981, pp.11-15.

Box, George E.P., and Jenkins, Gwilym M., "Time Series Analysis: Forecasting and Control,"revised edition, Holden-Day, San Francisco, 1976, pp.32-34.

Cadzow, James A., and Ogino, Koji, "Two-Dimensional Spectral Estimation,"IEEE Transactions on Acoustics, Speech, and Signal Processing, Vol. ASSP-29, No. 3, June 1981, pp. 396-401.

Jenkins,Gwilym M., and Watts, Donald G., "Spectral Analysis And Its Applications,"Holden-Day, San Francisco, 1968, pp.209- 257.

La Rocca, Anthony J. and Witte, David J.,"Handbook of the Statistics of Various Terrain and Water (Ice) Backgrounds from Selected U.S. Locations(U)," DTIC Technical Report Number 139900-1-X, January 1980, pp. 2-11 to 2-12.

Rapp,James R., "A Computer Model for Estimating Infrared Sensor Response to Target and Background Thermal Emission Signatures," Ballistic Research Laboratory, AMBRL-MR-03292, August 1983(AD\# B076976L), pp. 34-47.

Rapp,James R., private communication, December 1984. 
Rapp,James R. and Durfee,Gary L., private communication, 7 April 1987.

Sharma, Govind, and Chellapa, Rama, "Two-Dimensional Spectrum Estimation Using Noncausal Autoregressive Models,"IEEE Transactions on Information Theory, Vol. IT-32, No. 2, March 1986, pp. 268-275.

Strenzwilk, Denis F., Meredith, Michael P., and Federer, Walter T., "A Simple Mathematical Model for the Simulation of IR Backgrounds," in the Proceedings of The 33rd Conference of Design of Experiments in Army Research,Development, and Testing, May 1988, ARO Report 88-2, pp. 27-36. 


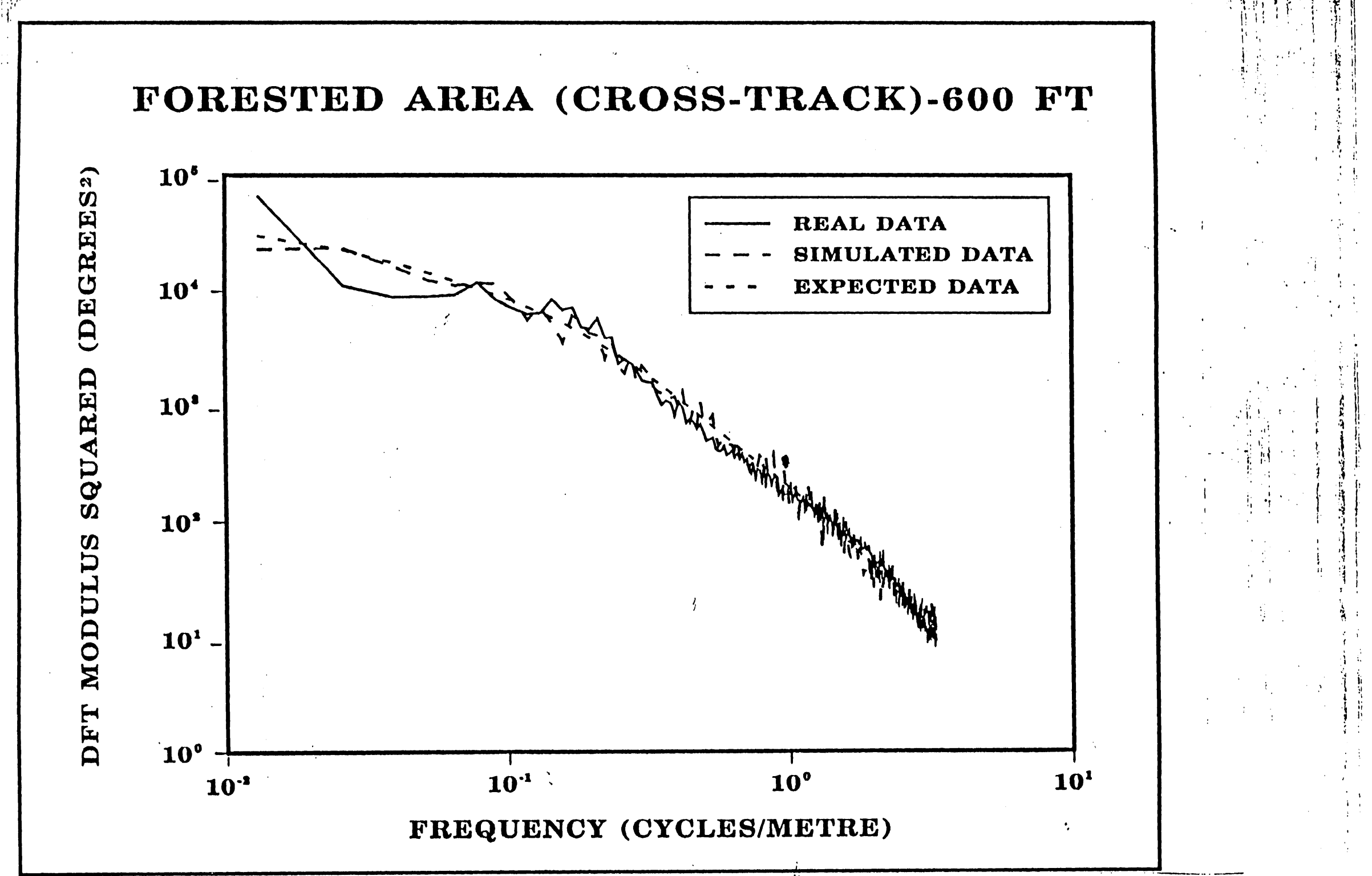

Figure 1. The cross - track energy spectra of the real, simulated, and expected data of a forested area at 600 -feet altitude are plotted. 


\section{FORESTED AREA (IN-TRACK)-600 FT}

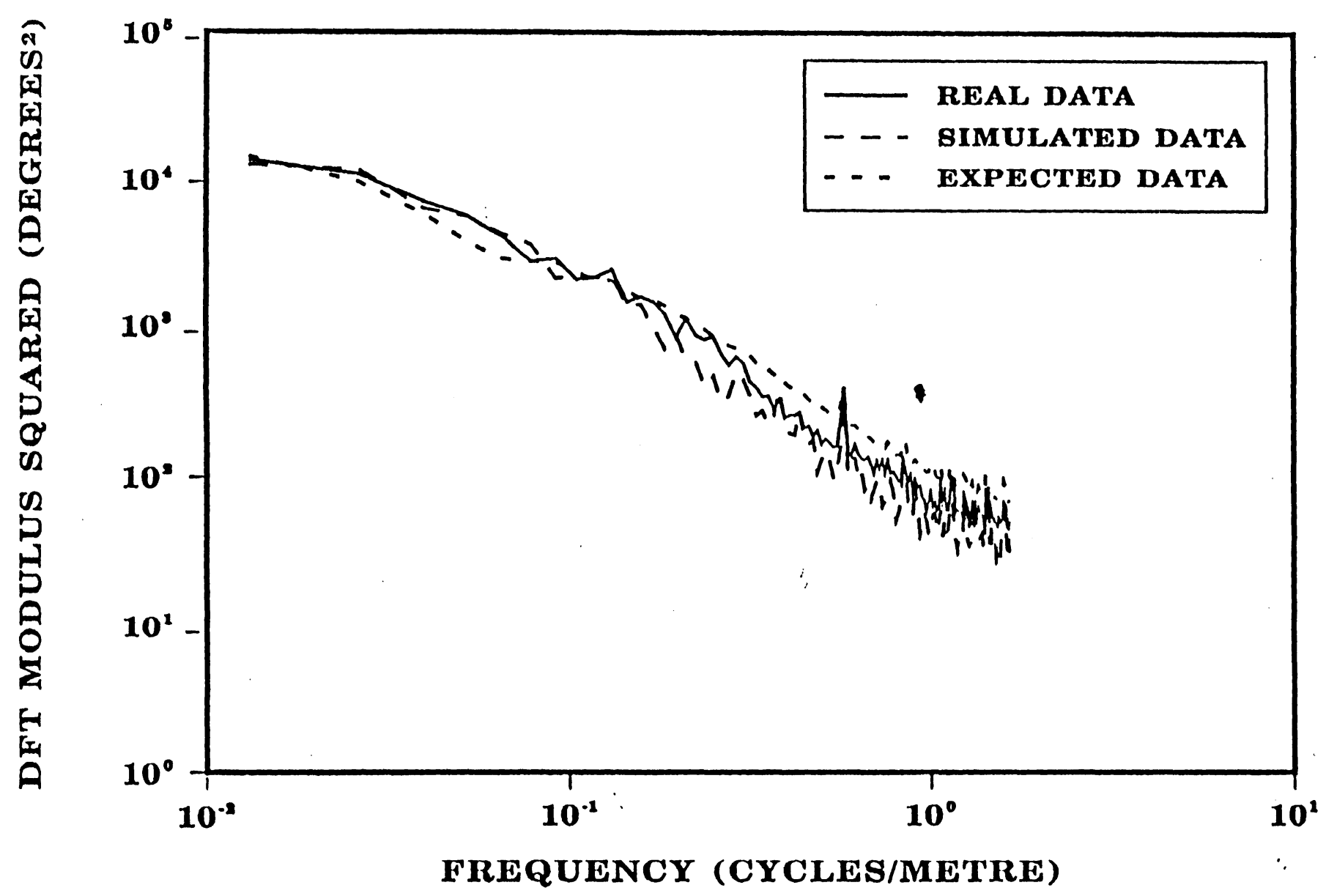

Figure 2. The in - track energy spectra of the real, simulated, and expected data of a forested area at 600 -feet altitude are plotted. 